\title{
Multipoint observation of the response of the magnetosphere and ionosphere related to the sudden impulse event on 19 November 2007
}

\author{
Antoni Segarra ${ }^{1, *}$, Masahito Nosé ${ }^{2}$, Juan José Curto ${ }^{1}$, and Tohru Araki ${ }^{3}$ \\ 1 Observatori de l'Ebre, (OE), CSIC - Universitat Ramon Llull, Roquetes 43520, Spain \\ *Corresponding author: asegarra@obsebre.es \\ 2 Data Analysis Center for Geomagnetism and Space Magnetism, Graduate School of Science, Kyoto University, \\ Kyoto 606-8502, Japan \\ 3 Polar Research Institute of China, Shanghai 200136, China
}

Received 17 October 2014 / Accepted 18 May 2015

\begin{abstract}
The aim of this study is to provide a complete scope of a magnetic sudden impulse (SI) event along its way through interplanetary space and the magnetosphere until its arrival to the ground. In our case, we chose the event of 19th November 2007 because of the availability of enough well-located spacecraft at that moment for our purpose. We have used a 16 spacecraft data set. We calculated the mass flux variation and the change in magnetic field components across the discontinuity. Thus, we identified the solar wind discontinuity as a shock. We also calculated the orientation of the solar wind shock front. Then, we examined the effects of the shock front propagation in detail. With this large data set, we obtained a global view of the travelling wave front and identified the effects of the compressional wave front. Thus, we determined in detail the shock front passing through the different parts of the magnetosphere. We described the compressional effects in the bow shock, the magnetosheath, and the magnetopause and we depicted the propagation inside the inner magnetosphere. Moreover, we used an extensive data set from magnetic observatories on the ground and so we studied the global distribution of the SI waveform. Finally, the comparison of the observational facts with those derived from the theoretical model showed a good consistency. On the basis of the waveforms and polarizations of this SI, we determined the location in latitude where ionospheric currents (ICs) changed their sense. And also, we related polarization at ground to polarization measured by GOES spacecraft.
\end{abstract}

Key words. Geomagnetism - Satellites (artificial) - Magnetosphere (inner) - Magnetosphere (outer) - Solar wind

\section{Introduction}

Geomagnetic sudden impulses (SIs) are produced by a sudden enhancement of solar wind dynamic pressure and are observed globally on the ground, with their amplitude and waveform changing greatly depending on latitude and local time. Since the global simultaneous occurrence of the SI phenomena with a clear onset time are the main characteristics distinguishing SIs from other magnetic field disturbances such as substorms and storms, SIs provide us with a fundamental understanding of a transient response of the magnetosphere and ionosphere to the solar wind. The interest in these phenomena led to the creation by the International Association of Geomagnetism and Aeronomy (IAGA) of a specific service called Service on Rapid Magnetic Variations (SRMV) with the aim of elaborating the official lists of SI events, and promoting the knowledge of them (Curto et al. 2007). Since 1975 this service is being maintained by the Ebro Observatory.

Due to the global simultaneous occurrence of the SI, it was one of the earliest phenomena to be studied on geomagnetism (for example, Adams 1892; Ellis 1892; Rodés 1922; Tanakade 1934). However, the different waveforms of SI, which depend on local time and latitude, were a great problem to understand the phenomena and even for detection and classification tasks (for example, Akasofu \& Chapman 1959; Matsushita 1960; Araki 1977).

Based on a large number of observational facts and previous studies, Araki (1994) developed a physical model for SI, which was able to explain the differences between waveforms observed at different latitudes and local time. Figure 1 summarizes the different waveforms of SI depending on latitude and local time. The content of the model is summarized as follows. The source of SI is a sudden increase of the dynamic pressure in the solar wind, when the discontinuity front of solar wind arrives to the magnetopause compressing the magnetosphere and propagates earthward in the dayside magnetosphere as a compression wave with a relevant hydromagnetic (HM) wave velocity, for example Tsurutani et al. $(2008,2014)$. The model decomposes the disturbed field of the SI waveform observed on the ground in two subfields, DL and DP (Araki et al. 2004). The subfield DL is a step-like function. The subfield DP field consists of two successive pulses with opposite senses. The subfield DL dominates at low latitudes and the subfield DP dominates at high latitudes (Araki et al. 2006). DL is produced mainly by the magnetopause current enhancement associated with the sudden compression of the magnetosphere. DP is further decomposed into two parts, $\mathrm{DP}_{\mathrm{PI}}$ and $\mathrm{DP}_{\mathrm{MI}}$, where $\mathrm{PI}$ and $\mathrm{MI}$ denote a preliminary impulse and a main impulse, 

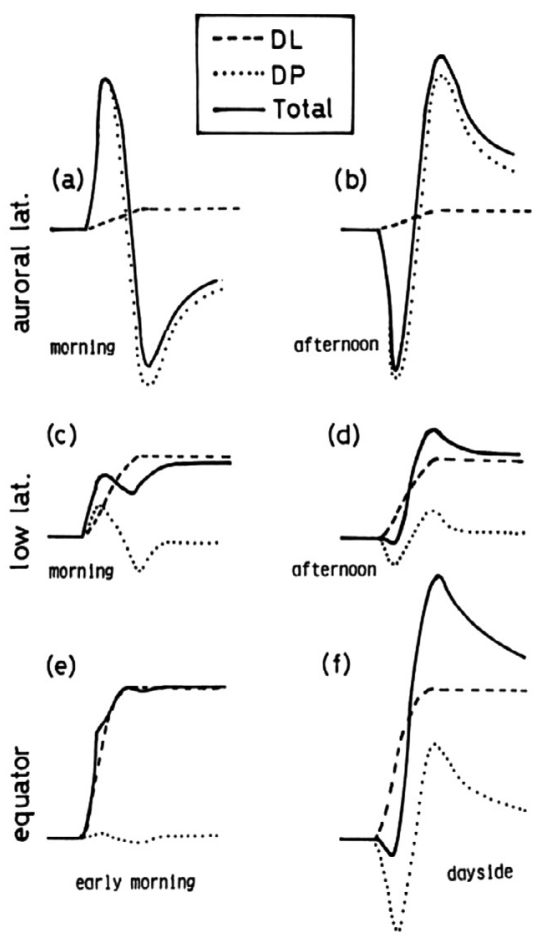

Fig. 1. Decomposition of SI disturbance field into DP and DL subfields depending on latitude and local time Araki (1994).

respectively. Both $\mathrm{DP}_{\mathrm{PI}}$ and $\mathrm{DP}_{\mathrm{MI}}$ are produced by field aligned currents (FACs) and resultant ionospheric currents (ICs) of polar origin. To summarize the different contributions to the disturbed field of SI, Araki (1994) introduced their relationship as follows:

$$
\mathrm{D}_{\mathrm{SI}}=\mathrm{DL}+\mathrm{DP}=\mathrm{DL}+\mathrm{DP}_{\mathrm{PI}}+\mathrm{DP}_{\mathrm{MI}}
$$

At high latitudes, where DP is dominant, the waveform of the SI in the $\mathrm{H}$ component consists of two successive pulses (PI and MI) with opposite senses. The amplitude of the pulses decreases with decreasing latitude. At low and mid latitudes, the waveform of the $\mathrm{H}$ component shows a more step-like function but the two-pulse structure with reduced amplitude is still superposed. At nightside equatorial latitudes, the waveform of the SI is mostly step-like but a small preliminary positive pulse (preliminary positive impulse, PPI) may be superposed upon the smooth rise of the SI. At the dayside equator, the two-pulse structure appears again, the waveform is similar to that in high latitude afternoon with a negative PI (preliminary reverse impulse, PRI). The amplitude of both pulses is enhanced compared with those at low latitudes. The enhancement rate is larger for PRI than for MI.

To explain these differences between waveforms, the proposed model assumes that a pair of ionospheric current vortices produced by field aligned currents moves from noon towards the night side at high latitudes when the preliminary impulse of SI is excited. The sense of the current rotation in the northern hemisphere is counter-clockwise (CCW) in the morning and clockwise (CW) in the afternoon under the low latitude side of current loops, but reverses under the higherlatitude side. When a compressional wave propagates towards the earth in the dayside magnetosphere, a dusk-to-dawn electric field along the wave front is transmitted along the lines of force to the northern polar ionosphere and produces a twin vortex ionospheric current system responsible for the preliminary impulse. As the compressional wave sweeps past the magnetosphere, the two vortices move from noon towards the dawnside and duskside. As a result of the motion, the magnetic field produced by the current vortices changes its direction at a fixed point on the ground. On the morningside the rotational sense of the horizontal magnetic vector is counterclockwise in the lower-latitude side of the centre of the vortex and clockwise in the higher-latitude side. On the afternoonside the rotational sense is clockwise in the lower-latitude side and counter-clockwise in the higher-latitude side.

Propagation of the compressional wave in the inner magnetosphere has been studied for many years; for example, Wilken et al. (1982) used multipoint observations of a sudden impulse taken by five spacecraft around the geosynchronous orbit to calculate the deformation of the SI wave front in the equatorial plane. Kokubun (1983) showed a clear dependence on local time for the amplitude of SI in the geosynchronous orbit. More recently, Keika et al. $(2008,2009)$ used more extensive multipoint observations in the inner magnetosphere and in the outer magnetosphere to study the decrease of the fast-mode wave speed in the plasmasphere relative to that in the outer magnetosphere, and global magnetic field variations in the inner magnetosphere in association with simultaneous substorm and magnetopause motion.

In this paper we present a case study of an SI event based on multipoint observations of a magnetic field. We used a large number of spacecraft observations that allowed us to describe how the compressional wave front propagated from the interplanetary magnetic field (IMF) to the magnetosheath, to the inner magnetosphere and to the ground. The reasons to choose this event were the large number of spacecraft available and, in particular, the preferred location of several of the spacecraft in or close to the magnetopause, providing us with a rare occasion of having data from these places just when an SI event occurred.

\section{Overview of the event}

We are interested in discussing an SI event and its propagation through the whole magnetosphere, with the same method used by Keika et al. (2008, 2009), multipoint spacecraft observations; we focused on magnetic effects, both in the magnetosphere and at the ground. As a first approach to the event, in Figure 2 we plotted data from the WIND spacecraft including the solar wind dynamic pressure $\left(P_{\mathrm{d}}\right)$, and the $Z$ component of the interplanetary magnetic field (IMF) $\left(B_{z}\right)$, as well as the SYM-H and AE indices for the 19th and 20th of November 2007. The SYM-H index indicates the symmetric disturbance field in H (Iyemori 1990) in a 1-min cadence and replaces Sugiura and Poros' hourly disturbed storm time or $D_{\text {st }}$ index (Sugiura \& Poros 1971; Wanliss \& Showalter 2006). SYM-H reflects the magnetic signature only for low latitudes, we plotted $\mathrm{AE}$ index to show magnetic signatures for high latitudes (Davis \& Sugiura 1966). Then, we identified and related different features of $P_{\mathrm{d}}$ and $B_{z}$ at the WIND location with the SYM-H and AE indices at ground, taking into consideration a 48-min propagation delay between the arrival of the perturbation at spacecraft and at the ground.

The first feature that we identified is marked with a dashed line at 1722 UT in Figure 2, and corresponds to a $P_{\mathrm{d}}$ enhancement of $2 \mathrm{nPa}$ at the WIND location. $P_{\mathrm{d}}$ continued to increase reaching a peak value of $12.3 \mathrm{nPa}$ at $2037 \mathrm{UT}$ in Figure 2 and then suddenly dropped from $9 \mathrm{nPa}$ to $2 \mathrm{nPa}$ at $2354 \mathrm{UT}$, as 
A. Segarra et al.: Multipoint observation of the magnetosphere response to a SI event

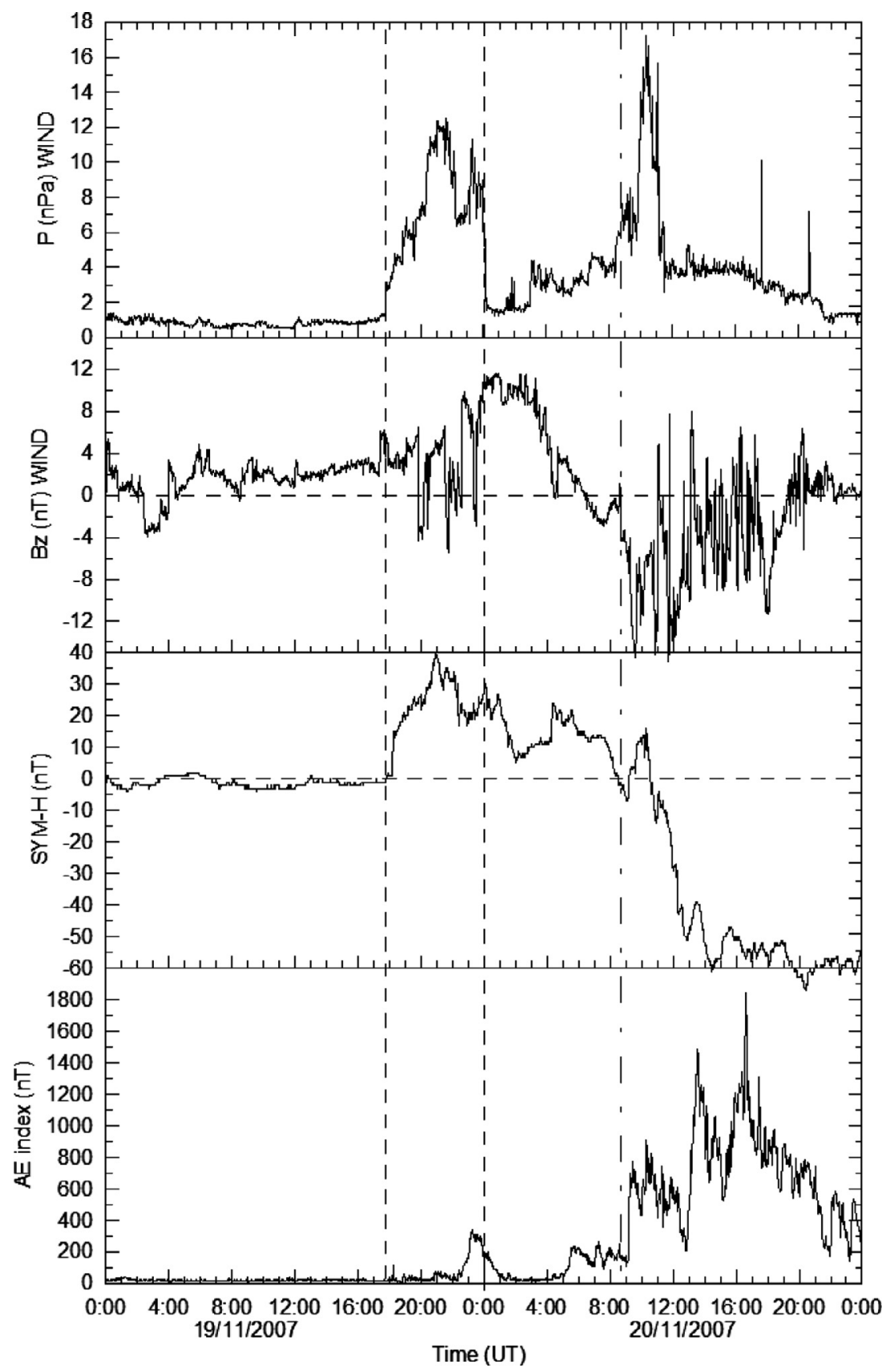

Fig. 2. Solar wind dynamic pressure and interplanetary magnetic field ( $Z$ component in GSM coordinates) measured by Wind spacecraft, SYM-H and AE indices measured at ground. Dashed lines mark an area that we can relate from Wind to ground that corresponds to an enhancement of $P_{\mathrm{d}}$ and SYM-H, the delay is due to their different locations. Dash-dotted line marks when $B_{z}$ becomes negative suddenly and then the main phase of the magnetic storm starts, AE suddenly increases.

indicated by a second dashed line in Figure 2. The SYM-H trace follows the same behaviour as $P_{\mathrm{d}}$, with a 48-min propagation delay sharply increasing by $15 \mathrm{nT}$ when $P_{\mathrm{d}}$ first increases. The second feature we identified, marked with a dot-dashed line in Figure 2, clearly corresponds to a sudden decrease in $B_{z}$, with a slope of $-4 \mathrm{nT} / \mathrm{min}$, which is followed by around 10-hour period of negative $B_{z}$. Then, on the ground, with a delay of $30 \mathrm{~min}$, the SYM-H started a decreasing evolution and $\mathrm{AE}$ index increases suddenly which corresponds to the main phase of a magnetic storm.

In Figure 3, we showed the solar wind parameters in more detail: IMF $\left(B_{x}, B_{y}, B_{z}\right)$, dynamic pressure $\left(P_{\mathrm{d}}\right)$, density $(N)$, and velocity $\left(V_{x}, V_{y}, V_{z}\right)$, observed by the WIND spacecraft.
There is a small enhancement of dynamic pressure at 1722 UT. Simultaneously, a rapid increase in $B_{z}$ is seen, and $V_{x}$ becomes more negative with a sudden increase of its magnitude. As a result of the arrival of the compressional wave to the magnetopause and the fact that at ground we observe an enhancement of the magnetic field, it is appropriate to define this propagation as a shock wave sweeping the whole magnetosphere.

\section{Spacecraft observations}

We used solar wind data from ACE, WIND, GEOTAIL, and THEMIS B. We also used data from the GOES, CLUSTER, 


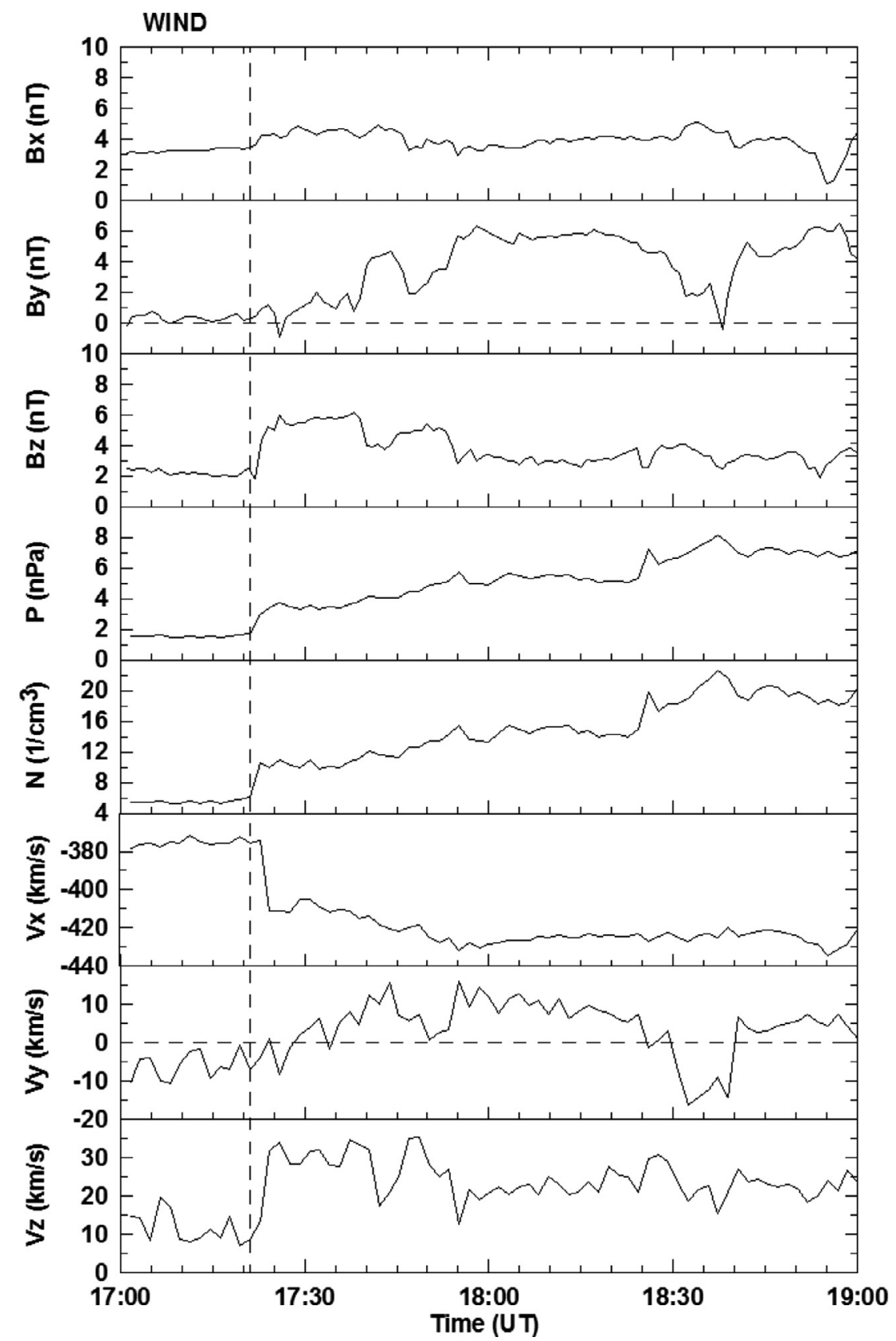

Fig. 3. Panel showing several solar wind parameters: three components of IMF, dynamic pressure, particle density, and three components of the solar wind velocity, measured by WIND spacecraft for 1700-1900 UT on 19 November 2007. Dashed line indicates the arrival of shock front.

THEMIS and LANL spacecraft in the magnetosphere. The data available provides a complete view of the event; from the IMF propagation, through different parts of magnetosphere, to the worldwide event at the Earth. Table 1 lists the positions of the spacecraft when the SI occurred.

\subsection{Solar wind observations}

For solar wind monitoring we used data from several instruments: the WIND Magnetic Field Investigation (MFI; Lepping et al. 1995) and 3-D Plasma and Energetic Particle Investigation (Lin et al. 1995), and the ACE Magnetic Field Experiment (MAG) instruments (Smith et al. 1998). We used data obtained by the Solar Wind Analyser (SWA) of the Comprehensive Plasma Instrumentation (CPI) (Frank et al. 1994) and the magnetic field measurements (MGF) system (Kokubun et al. 1994) on board the GEOTAIL spacecraft. Figure 4 shows the positions of the ACE, WIND, GEOTAIL and THEMIS B spacecraft in the $X Y$ and $X Z$ planes in GSM coordinates during the SI event.

In Figure 5 we present the IMF measured by ACE, WIND, GEOTAIL and THEMIS B spacecraft on 19th November 2007 at 1700-1900 UT. ACE, WIND and GOETAIL showed the same behaviour of IMF; an enhancement of total component, a clear increase of $B_{z}$ component and a smooth enhancement of $B_{x}$ and $B_{y}$ components. In the $B_{y}$ component there is a clear decrease, at around $1820 \mathrm{UT}$, for ACE spacecraft, also observed for WIND and GEOTAIL spacecraft (for GEOTAIL not shown in Fig. 5), which implies a large-scale structure of IMF. In order to distinguish whether the solar wind 
Table 1. Spacecraft positions at 1810 UT on the 19th of November 2007.

\begin{tabular}{lrrr}
\hline \hline Spacecraft & $X$-GSM (Re) & Y-GSM (Re) & Z-GSM (Re) \\
\hline ACE & 233.2 & -37.1 & 1.1 \\
Cluster-2 & 3.5 & -0.3 & -6.0 \\
Cluster-4 & 3.9 & -0.5 & -5.5 \\
Geotail & 26.7 & -3.4 & -3.8 \\
GOES-10 & 5.2 & 3.5 & 2.0 \\
GOES-11 & 4.6 & -4.5 & 1.3 \\
GOES-12 & 5.9 & 1.9 & 2.2 \\
LANL-01 & -1.2 & 6.5 & 0.0 \\
LANL-02 & -5.9 & 1.9 & -1.9 \\
LANL-89 & 3.8 & -5.2 & 1.0 \\
LANL-94 & 4.4 & 4.5 & 1.8 \\
LANL-97 & -3.2 & -5.5 & -1.5 \\
THEMIS-A & -4.2 & -13.6 & -2.1 \\
THEMIS-B & -9.7 & -29.4 & -4.6 \\
THEMIS-C & -0.1 & -16.1 & -1.6 \\
THEMIS-D & 1.6 & -9.6 & -0.5 \\
THEMIS-E & 0.7 & -10.7 & -0.9 \\
Wind & 235.5 & 88.0 & 1.2 \\
\hline
\end{tabular}
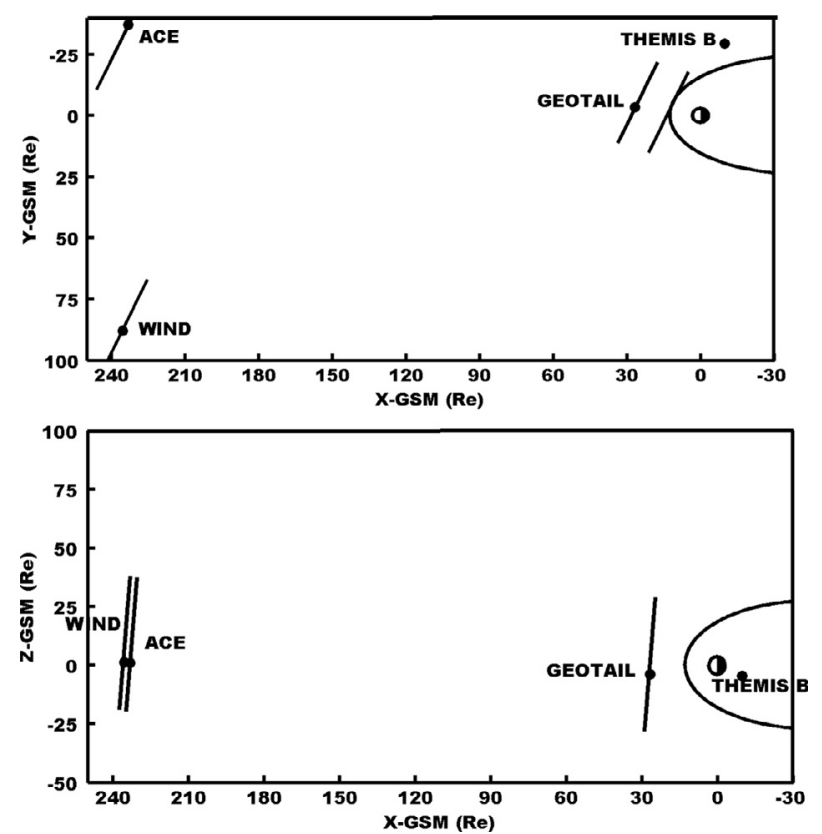

Fig. 4. Positions in $X Y$ and $X Z$ planes in GSM coordinates for ACE, WIND, GEOTAIL and THEMIS B spacecraft. Solid lines indicate the orientation of the solar wind shock front for each spacecraft. The orientation of the shock front when it arrives to the magnetopause is also drawn.

discontinuity shown in Figure 5 can be characterized as directional discontinuity, rotational discontinuity, tangential discontinuity, contact discontinuity or shocks (see Tsurutani et al. 2011, 2014 for more details), we examined solar wind data from ACE, WIND and GEOTAIL using the Rankine-Hugoniot (R-H) method (Vorotnikov et al. 2008). According to our R-H analysis the events fulfill the characteristics of a shock. It presents mass flux and magnetic field penetration across the discontinuity surface $\left(\Delta(\rho V)=2.6 \mathrm{nPa} ; \quad \Delta \vec{H}_{\mathrm{t}}=2.9 \mathrm{nT}\right.$; $\Delta H_{\mathrm{n}}=1.9 \mathrm{nT} ; \Delta H_{\mathrm{t}}=2.5 \mathrm{nT}$; where $\Delta$ indicates the change across the discontinuity, and the subscripts $\mathrm{n}$ and $\mathrm{t}$ denote normal and tangential components to the discontinuity surfaces). In the following we will refer to this discontinuity as shock front.

THEMIS B presents other behaviour than ACE, WIND and GEOTAIL spacecraft because it is located in the bow shock and presents a feature that we will discuss in more detail in Section 3.2.1. We determined the onset time of the shock front arrival for each spacecraft, and so we had four onset times; ACE at 1712 UT, WIND at 1722 UT, GEOTAIL at 1804 UT and THEMIS B at 1812 UT, as indicated by dashed lines in Figure 5. With these onset times, spacecraft locations and magnetic field values we calculated the inclination and speed of the shock front. We used the conservation of the normal vector before and after the shock front passing each spacecraft on the basis of the coplanarity theorem. The shock normal (n) is written in the magnetic coplanarity (MC) method (Colburn \& Sonett 1966; Tsurutani \& Lin 1985) as follows:

$$
\hat{n}= \pm \frac{\left(\overrightarrow{B_{1}}-\overrightarrow{B_{2}}\right) \times\left(\overrightarrow{B_{1}} \times \overrightarrow{B_{2}}\right)}{\left.\mid \overrightarrow{B_{1}}-\overrightarrow{B_{2}}\right) \times\left(\overrightarrow{B_{1}} \times \overrightarrow{B_{2}}\right) \mid},
$$

where $\mathbf{B}$ is the magnetic field vector and subscripts 1 and 2 refer to the quantities ahead and behind the shock. Rapid fluctuations in the magnetic field recorded by spacecraft following the passage of the shock front made it difficult to take a unique value of $\mathbf{B}$ after the SI, and so we took an average over $2 \mathrm{~min}$. For the event, using average values, the $\mathbf{n}$ vector did not have exactly the same value for every spacecraft. So, on the basis of the locations and onset times of each spacecraft and with a 3D propagation model, we looked for a unique planar SI front which was the one most coherent with the whole data set. Proceeding in this way - first obtaining a shock normal for each spacecraft and then applying a $3 \mathrm{D}$ propagation model - we concluded that the unique coherent planar normal direction was $(\phi, \theta)=\left(155^{\circ}, 2^{\circ}\right)$, where $\phi$ and $\theta$ are the longitude and latitude in GSM coordinates. In Figure 4, in addition to the position of the spacecraft, we drew the orientation of the solar wind shock front with solid lines.

Using the shock normal direction calculated above and the onset time difference between each spacecraft, we can calculate the normal component of the shock velocity on the basis of mass flux conservation,

$$
V_{\text {Shock }}=\frac{\left(\rho_{2} \vec{V}_{2}-\rho_{1} \vec{V}_{1}\right) \cdot \hat{n}}{\rho_{2}-\rho_{1}},
$$

where $\rho_{1}$ and $\rho_{2}$ are the upstream and downstream solar wind mass density, respectively. The shock velocity was $V_{\text {Shock }}=540 \mathrm{~km} / \mathrm{s}$.

\subsection{Magnetosphere observations}

For observations in the magnetosphere, we used magnetic field data from GOES 10, 11 and 12, and THEMIS A, C, D and E spacecraft. Magnetic field data were obtained by Fluxgate Magnetometers (FGM; Auster et al. 2008). The four CLUSTER spacecraft (Balogh et al. 1997) carried Fluxgate Magnetometers (FGM) that provided 4-s magnetic field data. The Synchronous Orbit Particle Analyser (SOPA; Belian et al. 1992) on board the LANL spacecraft measured electrons with energies from $50 \mathrm{keV}$ to $26 \mathrm{MeV}$. We used 10-s electron data from five LANL 01, 02, 89, 94 and 97 spacecraft. In Figure 6 we plotted the locations of all the spacecraft. Moreover, we plotted the location of the first impact of the wave front 


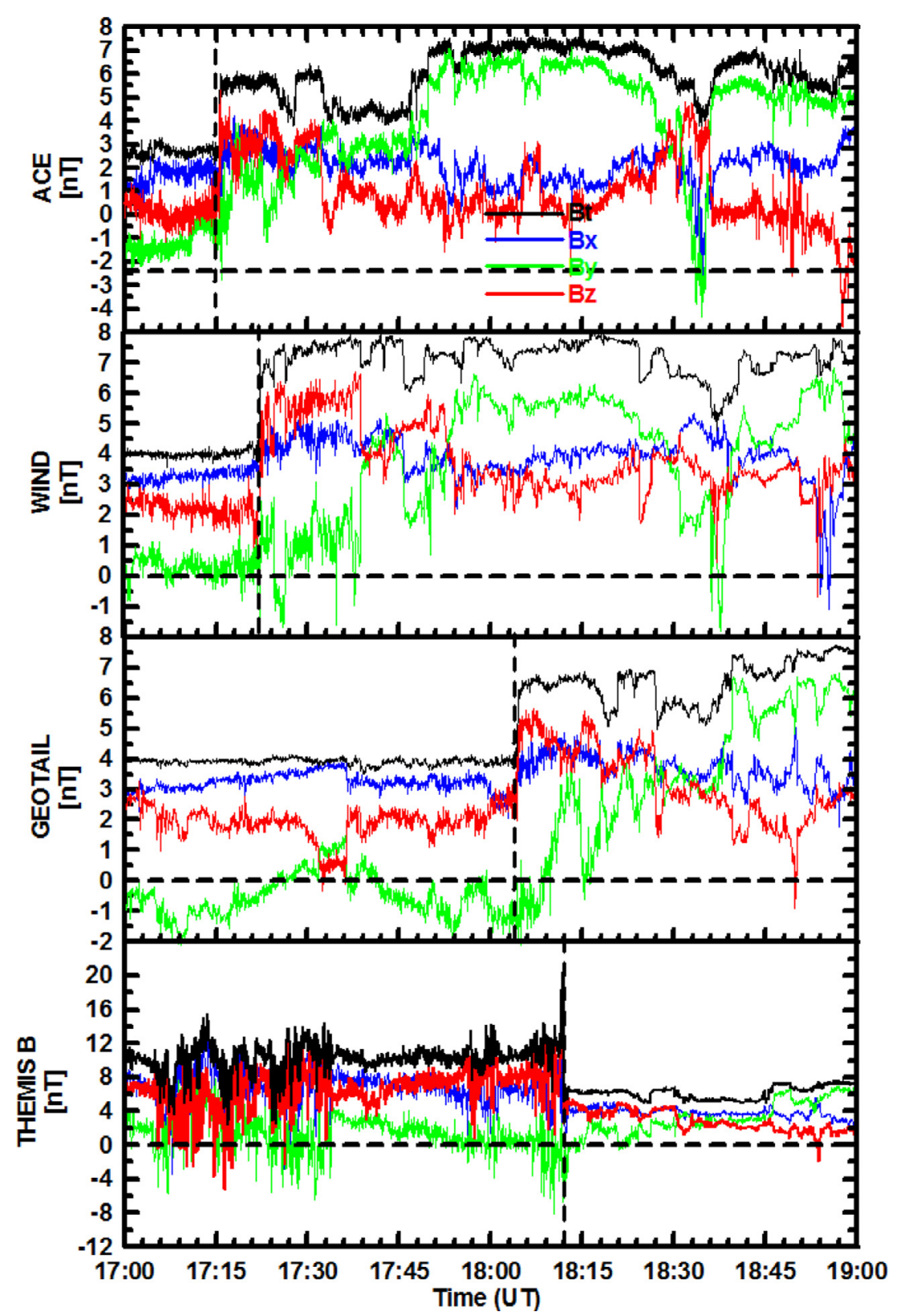

Fig. 5. Interplanetary magnetic field observed by spacecraft ACE, WIND, GEOTAIL and THEMIS B. $X$ component in blue, $Y$ component in green, $Z$ component in red and total component in black line. Dashed lines indicate the arrival time of the shock front.

with the magnetopause according to their inclination - calculated beforehand - and the position of the magnetopause, calculated from the value of solar wind pressure (Shue et al. 1998).

\subsubsection{Magnetosheath observations}

Taking advantage of the location of the THEMIS B spacecraft, in the magnetosheath, and the location of the THEMIS $\mathrm{C}$ spacecraft, close to the magnetopause, we can discuss how these parts of the magnetosphere respond to an enhancement of solar wind dynamic pressure. Figure 7 presents the magnetic field variation from 1500 to 2000 UT observed at GEOTAIL, upstream of the bow shock; THEMIS B, in the magnetosheath; THEMIS $\mathrm{C}$, in the magnetosheath close to the magnetopause; and THEMIS E inside the magnetosphere. It has three panels, one for each component of the magnetic field for each spacecraft. As the spacecraft were located in different regions, by comparing the different behaviours of the IMF $B_{z}$ in pairs, we can identify how and when the shock front compressed the different parts of the magnetosphere. First we compared THEMIS B and GEOTAIL. Both recorded a quiet magnetic field for 1500-1555 UT, in all three components, but the values of THEMIS B were more than the double of the values recorded by GEOTAIL, about $3.7 \mathrm{nT}$ for GEOTAIL $B_{x}$ component and $9.5 \mathrm{nT}$ for THEMIS B $B_{x}$ component. For $B_{y}$ and $B_{z}$ components, GEOTAIL has a value of about $2 \mathrm{nT}$, and THEMIS B has a value of about $5 \mathrm{nT}$. According to the location of the magnetopause calculated on the basis of solar wind pressure, we assumed that this value from THEMIS B corresponded to that of the magnetosheath magnetic field. After that, but still before the SI, from 1555 to 1810 UT, THEMIS B presented a disturbed field, which was consistent with oscillations also observed in GEOTAIL, as we commented above, and IMF showed a large-scale structure. At 1812 UT, we can see the wave front crossing. At that moment, the magnetosheath suffered a compression and THEMIS B had the same 
A. Segarra et al.: Multipoint observation of the magnetosphere response to a SI event
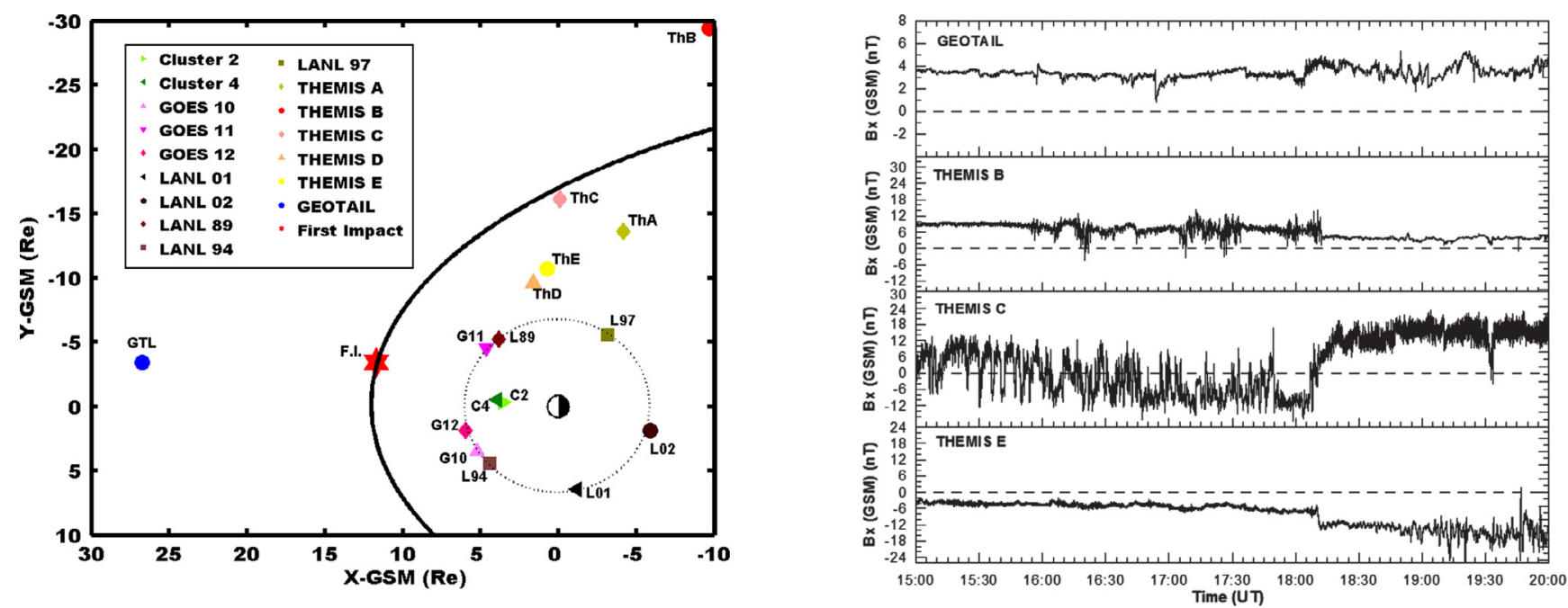

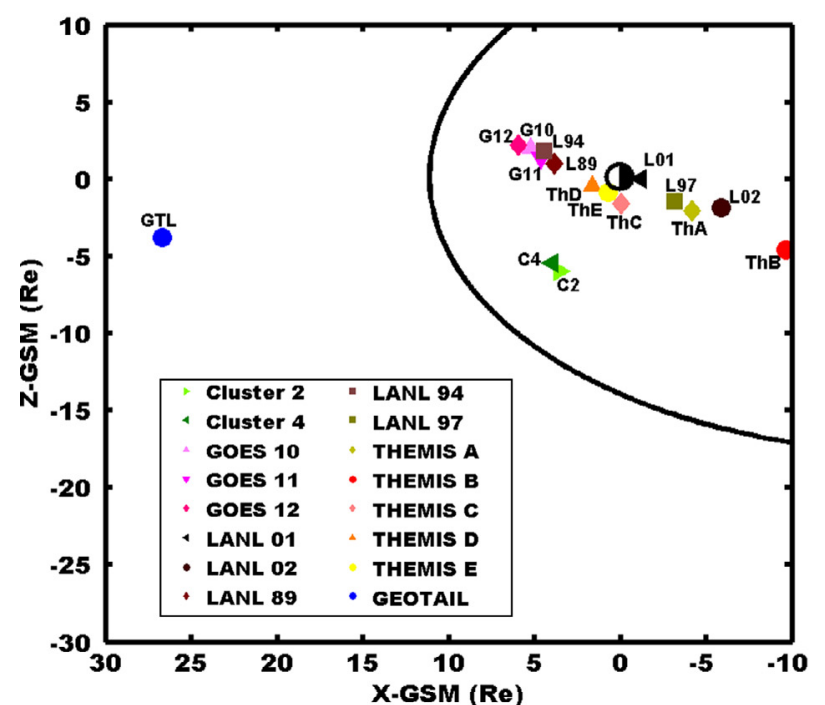

Fig. 6. Positions in $X Y$ and $X Z$ planes in GSM coordinates for GEOTAIL, CLUSTER, GOES, LANL and THEMIS spacecraft in the inner magnetosphere. We use a short name for each spacecraft. We plot the geosynchronous orbit with a dashed line. Symbol $(*)$ shows the point of the first impact of the wave front to the magnetopause.

values as GEOTAIL which monitored the IMF. We assumed that THEMIS B had suffered a bow shock crossing. Also around 1930 UT, THEMIS B saw the same magnetic structure as GEOTAIL did, with the clearest features about this appearing in the $Y$ component.

THEMIS $\mathrm{C}$ was located close to the magnetopause, and during the period 1500-1810 UT, it experienced a disturbed field in all three components. When the magnetic field signal was positive, in $X$ and $Y$ components, these values were in agreement with the magnetosheath values, like those of THEMIS B. And when the magnetic field signal was negative, these values were in agreement with values recorded by THEMIS E, located inside the magnetosphere. During this period, the $Z$ component of THEMIS B always remained positive. Then we assumed that these signal oscillations corresponded to magnetopause oscillations recorded by THEMIS C. After the
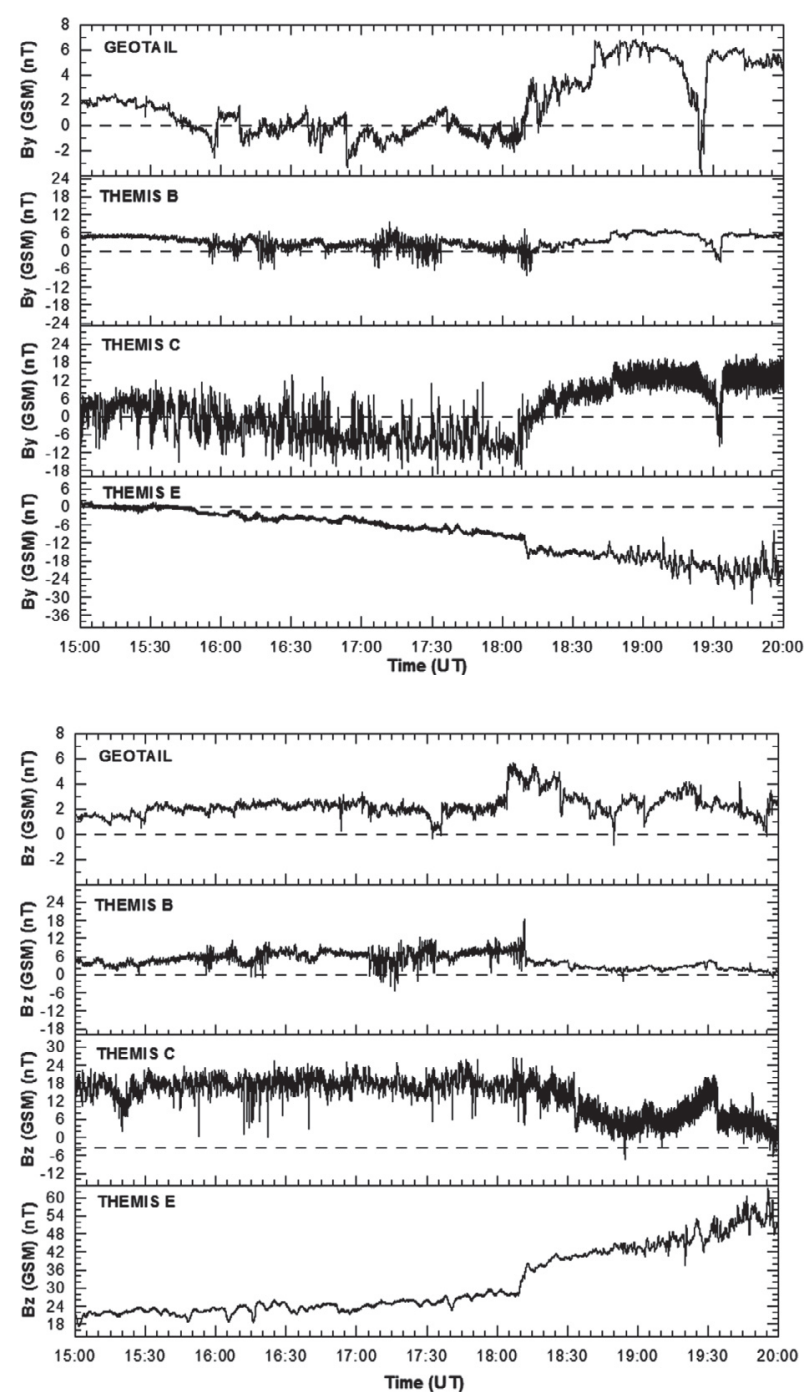

Fig. 7. Magnetic field (panel 1) $X$ component (panel 2) $Y$ component and (panel 3) $Z$ component in GSM coordinates as measured by the four spacecraft: GEOTAIL, located upstream of the bow shock; THEMIS B, located in the magnetosheath; THEMIS C, located close to the magnetopause; and THEMIS E, located in the inner magnetosphere. 


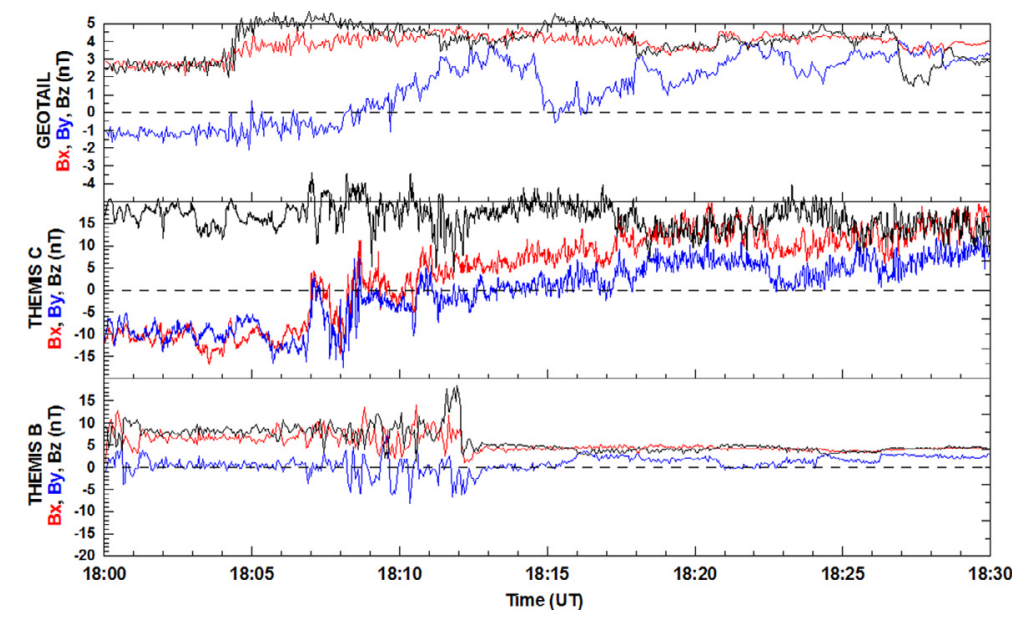

Fig. 8. $X, Y$ and $Z$ components of the IMF measured by the GEOTAIL, THEMIS B and C spacecraft for 1800-1830 UT on 19 November 2007 .

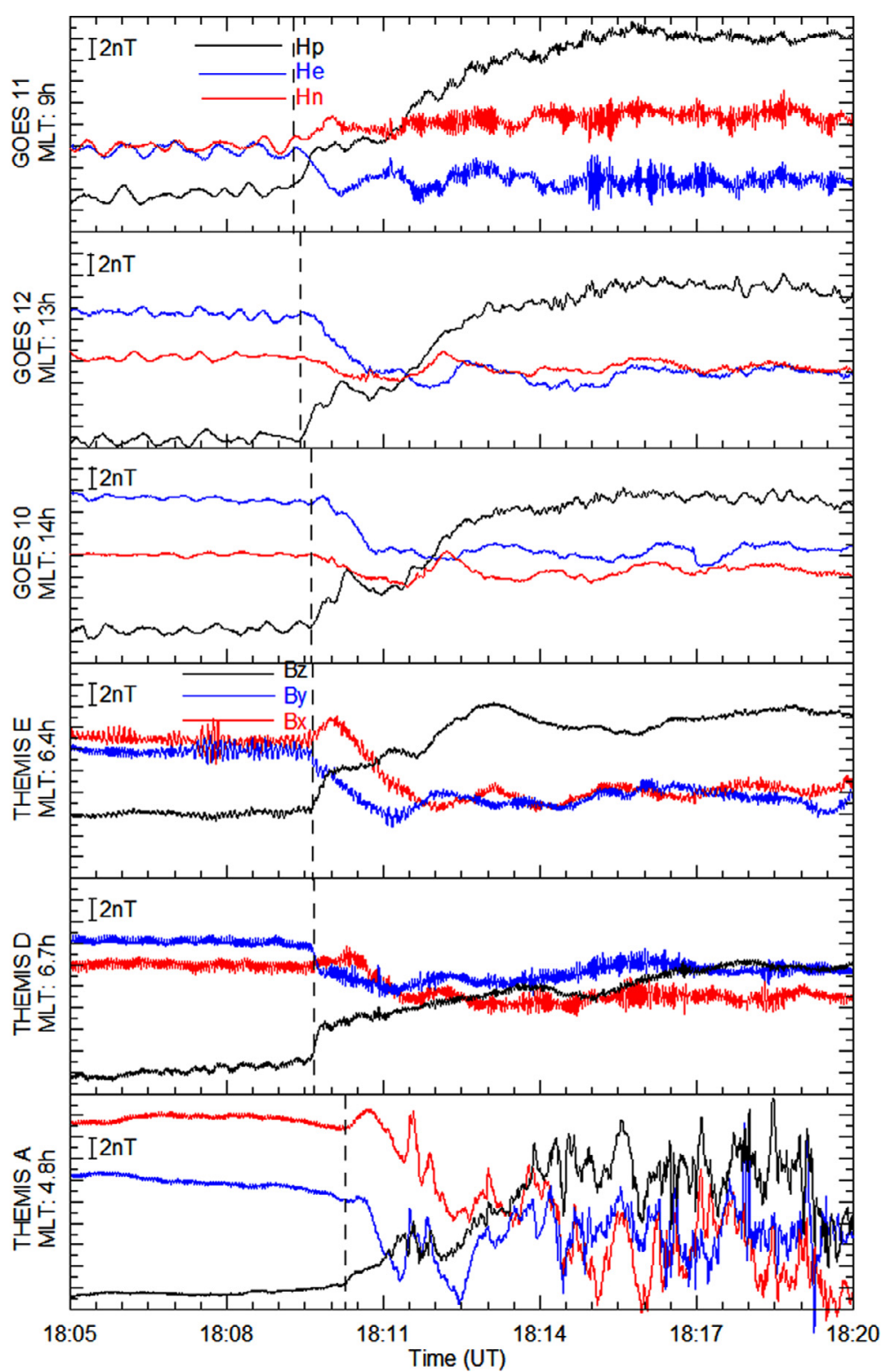

Fig. 9. Magnetic field observed at spacecraft located in the inner magnetosphere. For the GOES spacecraft, magnetic field is given in the direction parallel $\left(H_{\mathrm{p}}\right)$, earthward $\left(H_{\mathrm{e}}\right)$ and normal $\left(H_{\mathrm{n}}\right)$ to magnetic field lines. For the THEMIS spacecraft, magnetic field is given in the $X\left(B_{x}\right), Y\left(B_{y}\right)$ and $Z\left(B_{z}\right)$ directions in GSM coordinates. Parameters are plotted for 1800-1830 UT 19 November 2007. Dashed lines indicate the SI onset times. 
A. Segarra et al.: Multipoint observation of the magnetosphere response to a SI event

Table 2. Onset time, rise time, change in amplitude, magnetic latitude, magnetic local time and distance to the Earth for each spacecraft in the inner magnetosphere.

\begin{tabular}{|c|c|c|c|c|c|c|}
\hline Spacecraft & 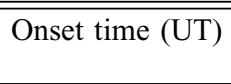 & Rise time (s) & $\begin{array}{c}\text { Change in } \\
\text { amplitude (nT) }\end{array}$ & $\begin{array}{c}\text { Magnetic } \\
\text { Latitude }\left({ }^{\circ}\right)\end{array}$ & $\begin{array}{c}\text { Magnetic } \\
\text { local time (h) }\end{array}$ & Radii (Re) \\
\hline GOES-11 & 18:09:09 & 34.2 & 3.8 & 4.5 & 9 & 6.6 \\
\hline GOES-12 & $18: 09: 23$ & 48.6 & 5.6 & 10 & 13 & 6.6 \\
\hline GOES-10 & $18: 09: 36$ & 41.7 & 5.4 & 4.5 & 14 & 6.6 \\
\hline THEMIS-E & $18: 09: 38$ & 15 & 2.9 & -5.0 & 6.4 & 10.7 \\
\hline THEMIS-D & $18: 09: 39$ & 8 & 2.6 & -3.3 & 6.7 & 9.7 \\
\hline THEMIS-A & $18: 10: 13$ & 17.8 & 1.3 & -8.6 & 4.8 & 14.4 \\
\hline
\end{tabular}

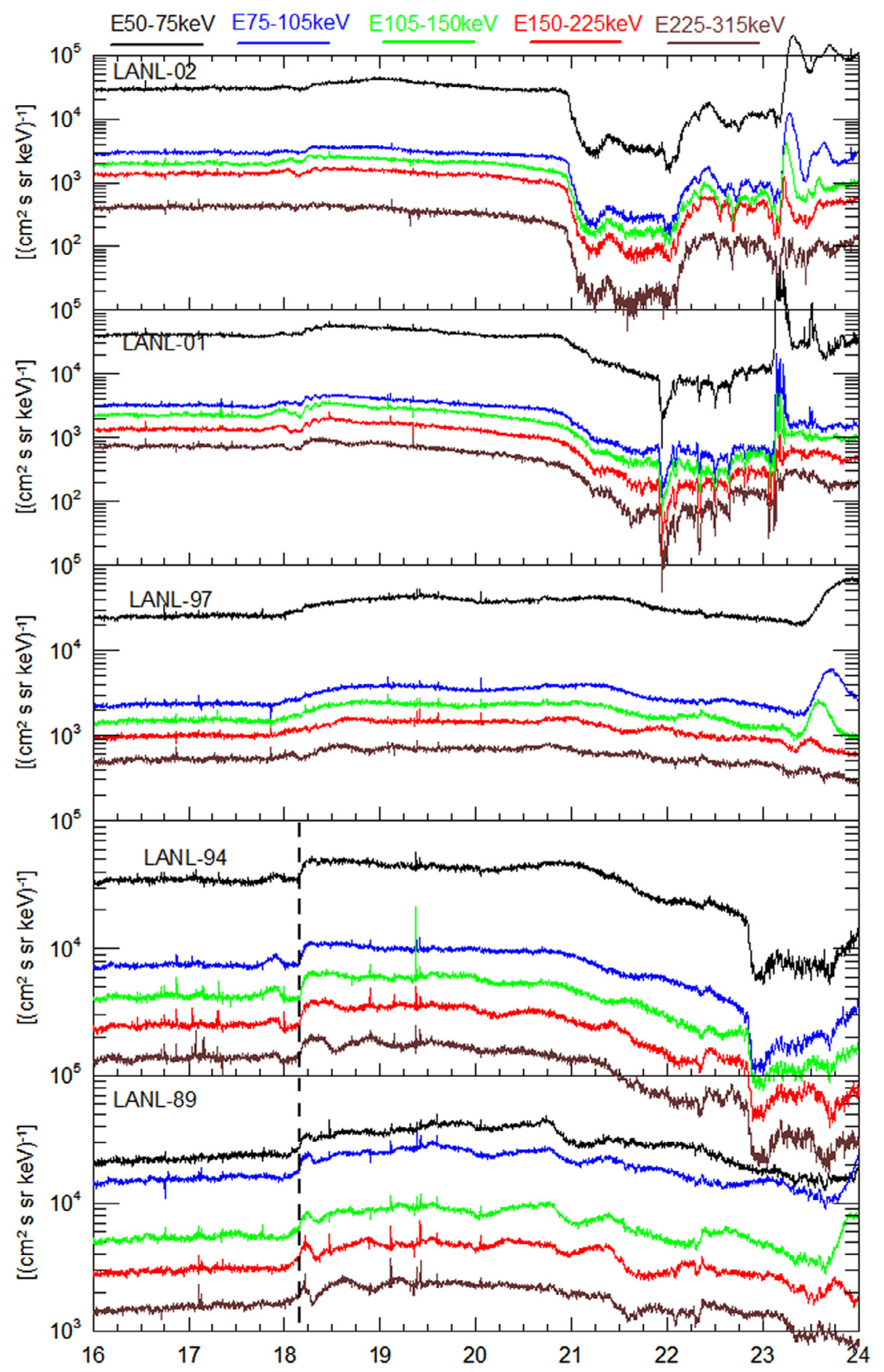

Fig. 10. Observations of the energetic electron flux measured by LANL for 50-75 keV (black line), 75-105 keV (blue line), 105-150 keV (green line), $150-225 \mathrm{keV}$ (red line) and $225-315 \mathrm{keV}$ (brown line). The dashed line at 1809 UT indicates the SI onset time which is clearly seen in LANL 89 and 94. For the other LANL spacecraft it is difficult establish a clear onset time, if it exists. 


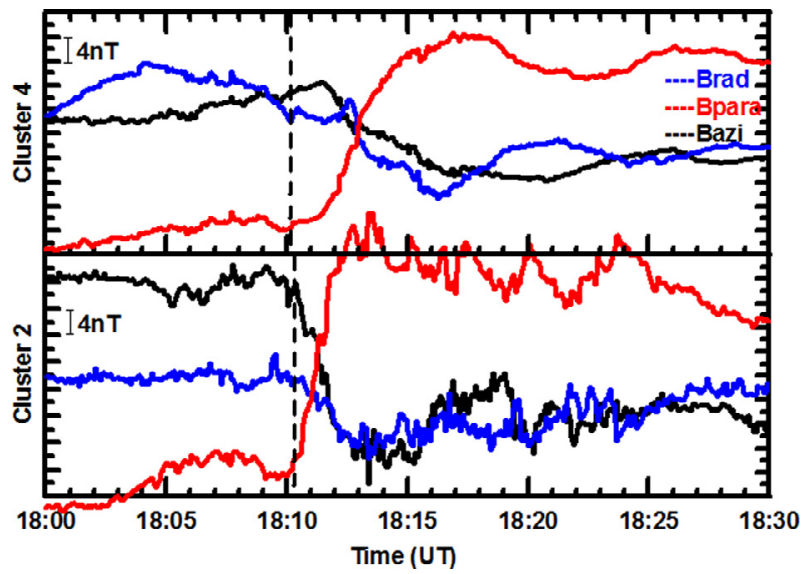

Fig. 11. Magnetic field observed at spacecraft located in the inner magnetosphere. For the CLUSTER 2 and 4 spacecraft, magnetic field is given in the direction radial $\left(B_{\mathrm{rad}}\right)$, parallel $\left(B_{\mathrm{para}}\right)$ and azimuthally $\left(B_{\text {azi }}\right)$ to magnetic field lines. Dashed lines indicate SI onset time for each spacecraft.

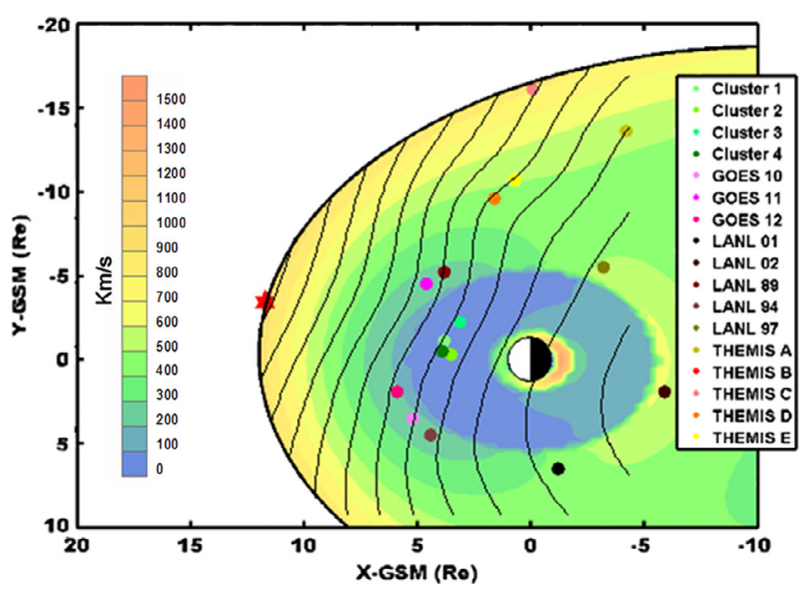

Fig. 12. In solid dark lines, we present wave front propagation into the inner magnetosphere, as calculated on the basis of onset arrival times. In contour colours, we show Alfven velocity results according to fast-mode propagation calculations.

shock front passing, THEMIS C recorded values showing structures with the same behaviour as in the magnetosheath; also, the clearest features appear in the $Y$ component. Summarizing, with these four spacecraft we could identify the shock front passing and the compression of the magnetosheath and magnetopause.

In order to determine more precisely the onset times, we used an expanded time representation. In Figure 8, we showed the magnetic field recorded by GEOTAIL, THEMIS B and C, with an expanded time scale (from 1800 to 1830 UT). THEMIS B was in the magnetosheath before 1812 UT. A sharp increase of the $B_{z}$ component just after 1811 UT indicated the arrival of the interplanetary shock. The sharp $B_{z}$ decrease around 1812 UT was due to the bow shock crossing because THEMIS B was in interplanetary space after 1812 UT. GEOTAIL was located in interplanetary space and the $B_{z}$ component suddenly increased by $2.5 \mathrm{nT}$ at $1804 \mathrm{UT}$. For THEMIS B, the amplitude of the shock was around $12 \mathrm{nT}$, about five times greater than for GEOTAIL. These facts showed how different the magnetic activity was in these regions. THEMIS $\mathrm{C}$ was located near the magnetopause and it was really difficult to determine a clear onset time for the shock front. This was because the magnetic field was disturbed and the amplitude of the shock was not very high, so the arrival of the shock could be confused with an oscillation of the disturbed field.

As a summary, we can observe interplanetary shock propagation on the basis of different onset times at each spacecraft, for ACE at $1712 \mathrm{UT}$, for WIND at $1722 \mathrm{UT}$, for GEOTAIL at 1804 UT and for THEMIS B at 1812 UT.

\subsubsection{The inner magnetosphere}

As we commented above, Figure 6 shows the location of the spacecraft inside the magnetosphere. From the inclination and velocity data calculated in Section 3.1, we determined the first arrival of the wave front at the magnetopause at 1807 UT. The position of the first arrival was $X=11.7 \mathrm{Re}$, $Y=-3.4 \mathrm{Re}$ and $Z=2.3 \mathrm{Re}$. Figure 9 shows the magnetic field values recorded by the THEMIS and GOES spacecraft inside the magnetosphere. Data are ordered according to the arrival times of the wave front at the spacecraft, between 1805 and 1820 UT. For all six spacecraft an enhancement of the magnetic field appeared; in the Hp component (parallel to dipole field) for the GOES spacecraft, and in the $B_{z}$ component (GSM coordinates) for the THEMIS spacecraft. GOES components $H_{\mathrm{e}}$ (Earthward magnetic flux) clearly decreased and $H_{\mathrm{n}}$ (eastward magnetic flux) smoothly increased for GOES 11 and clearly decreased for GOES 10 and 12. THEMIS $B_{x}$ component smoothly increased and after that clearly decreased, and THEMIS $B_{y}$ clearly decreased. In Table 2, we show onset time, rise time and the change in amplitude of the magnetic field observed at the position of each spacecraft caused by the arrival of the shock front. For every spacecraft an enhancement was clearly seen.

An important aspect helping to relate GOES observations with ground observations arose in the polarization of the magnetic signature; for GOES $11, H_{\mathrm{e}}$ decreased and $H_{\mathrm{n}}$ increased, which was equivalent to a clockwise polarization. And for GOES 10 and 12, located in the afternoon sector, $H_{\mathrm{e}}$ decreased and $H_{\mathrm{n}}$ decreased, which was equivalent to a counter-clockwise polarization.

In Figure 10 particle data from the LANL are shown. We can only determine a clear onset time, dashed lines in Figure 10, of the arrival shock front for spacecraft located at dayside, LANL 89 and 94. For the other spacecraft, the enhancement, if it exists, is so smooth that the onset could not be determined. The explanation for this is as follows. To accelerate particles there should be a large electric field, which is induced by temporal changes of the magnetic field. On the dayside, temporal changes are rather sharp or steep so that a strong electric field is created and can accelerate particles. However, on the night side which is far from the shock arrival point, magnetic field changes become less steep, and only a small electric field is induced and no clear particle flux enhancement is observed.

Figure 11 shows magnetic data from the CLUSTER spacecraft. Magnetic field is given in the direction radial $\left(B_{\mathrm{rad}}\right)$, parallel $\left(B_{\text {para }}\right)$ and azimuthally $\left(B_{\text {azi }}\right)$ to magnetic field lines. CLUSTER 2 and 4 can be used to determine the onset time. We investigated the response of the inner magnetosphere to the magnetospheric compression caused by the interplanetary shock around 1810 UT on 19th November 2007. Taking into 
A. Segarra et al.: Multipoint observation of the magnetosphere response to a SI event

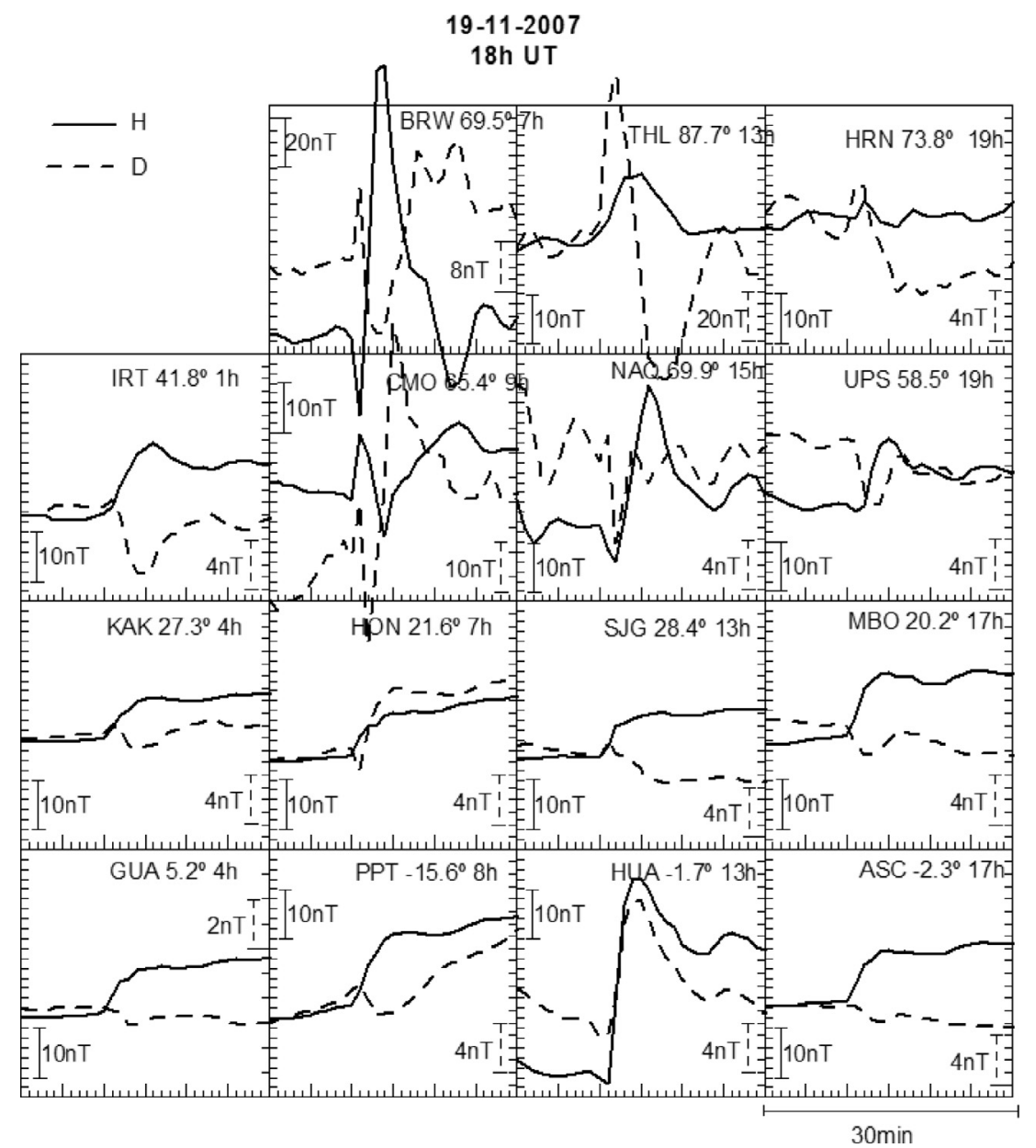

Fig. 13. Summary of SI recorded at ground. Rows are sorted from low to high magnetic latitude. Columns are sorted according to the magnetic local time. Each panel shows magnetic field, H component (solid line) and D component (dashed line), between 1800 UT and 1830 UT for each observatory.

consideration the time lag between each pair of neighbouring spacecraft, we estimated the propagation speed in the inner magnetosphere at around $900 \mathrm{~km} / \mathrm{s}$; higher than the shock speed in the solar wind.

With onset times of all these spacecraft inside the magnetosphere we had enough points to make a grid and use an interpolation method based on regression analysis to calculate the propagation of the wave front (dark lines in Fig. 12). Coloured contours in Figure 12 indicate the Alfven velocity according to fast-mode propagation calculations using a model by Tsyganenko (1989) for the magnetic field, and following the empirical model of Carpenter \& Anderson (1992) for the mass density:

$$
\begin{gathered}
n_{\text {plasmasphere }}=200\left(\frac{5}{r}\right)^{4} m, \\
n_{\text {plasmatrough }}=0.6\left(\frac{10}{r}\right)^{4} \mathrm{~m}, \\
n_{\text {plasmasheet }}=\frac{0.6}{(1-\exp (-r 12))} \mathrm{m},
\end{gathered}
$$

where $n$ is the mass density in $\left(\mathrm{kg} \mathrm{cm}^{-3}\right), r$ is the radial distance in $\left(\mathrm{R}_{\mathrm{E}}\right)$ and $m$ is the proton mass. Equation (4) is valid for the plasmasphere, Eq. (5) for the plasmatrough and
Eq. (6) for the plasma sheet. A deformation of the compressional wave front appears in the central part of the magnetosphere where we had most of the observation points. It is consistent with Alfven speed variations due to different density areas in the magnetosphere. The deformations on the night side were less reliable and were probably due to methodological problems at boundaries where we did not have many observation points.

\section{Ground data}

We analysed a complete 1-min data set from ground observatories to describe the behaviour of this SI event. We were interested in the waveform and its polarization compared with those derived from Araki's model. A comparison of quantitative results of the model with the data presented in the paper is difficult because the model predicts and explains fairly well how are the different waveforms of SI depending on latitude and local time, but, as stated in that paper, quantitative determinations of the amplitudes would need a precise estimation of the ionospheric conductivity and FAC intensities.

Figure 13 shows $\mathrm{H}$ and $\mathrm{D}$ components of the magnetic field for 15 magnetometer stations sorted by their magnetic latitude and magnetic local time. Regarding the D component, the dashed line in Figure 13, stations plotted in the same row, 


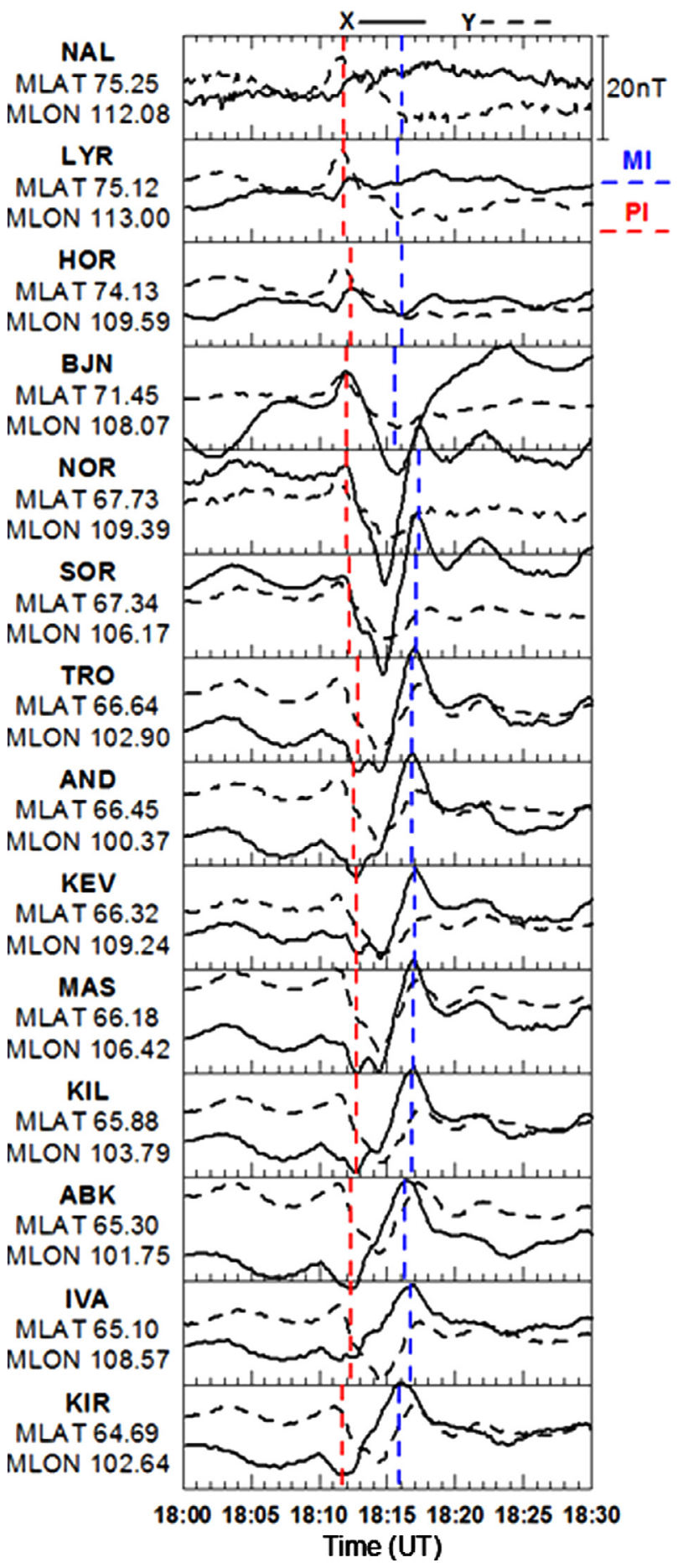

Fig. 14. Magnetograms from IMAGE array magnetometers, ordered from high to low magnetic latitude. Vertical dashed line in red indicates preliminary impulse, PI, and vertical dashed line in blue indicates main impulse, MI.

KAK and HON, present a small increase in the D component, and SJG and MBO present a small decrease. Stations plotted in another row, such as CMO and NAQ, showed a reversed behaviour with respect to the observatories showed in the under row. We can explain the waveform changes on the basis of Tsunomura's model (Tsunomura \& Araki 1984). This model proposed a distribution of the ionospheric currents based on a realistic conductivity distribution. This model predicted that the North-South component of the ionospheric currents should

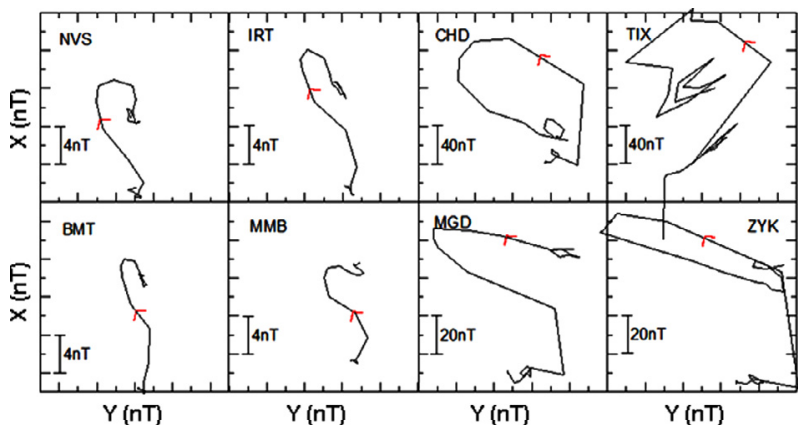

Fig. 15. Polarization diagram representing the temporal evolution of the magnetic vector for magnetic observatories located in the meridian plane including the point of the first impact of the wavefront to the magnetopause. This polarization is the result of the synchronic variation of its components. Thirty minutes around the SI onset time, from 1800 to 1830 UT, are plotted. In every panel, $X$ component is the ordinate and $Y$ component is the abscissa. In the four panels on the left the polarization is linear. In the four panels on the right the polarization is counter-clockwise.

change its sign four times a day in a latitude region between $30^{\circ}$ and $70^{\circ}$ and the largest amplitude of this component should happen in the morning hours. Thus, the $\mathrm{D}$ geomagnetic component should present a collection of boundaries where its behaviour must change depending on local magnetic time and latitude.

Solid lines in Figure 13 represent the $\mathrm{H}$ component of the magnetic field which can be compared with the prediction of Araki's model shown in Figure 1. As we explained in Section 1, subfield DL has a waveform characterized by a step-like function, and is dominant with respect to the DP subfield for stations located at low and mid latitudes. Such a waveform is predominant at the low and mid latitude station plotted in the bottom two rows of Figure 13. The step-like function dominates for all stations with the exception of Huancayo (HUA) station $\left(-1.7^{\circ}\right.$ Glat.). At HUA the DL and DP subfields were superimposed and we can observe a PRI (preliminary reverse impulse), this is because the ionospheric vortex extends its effects until equatorial latitudes at dayside. Also, another important characteristic at Huancayo was the large amplitude of the SI, the largest one at any low latitude station, about $30 \mathrm{nT}$ for HUA and around $10 \mathrm{nT}$ for ASC, GUA or PPT. It was produced by the enhancement of the electric conductivity in that area (equatorial electrojet).

In Figure 13, with stations in the same row as IRT, CMO, NAQ and UPS, we can observe how the waveforms vary depending on the magnetic local time. Near the midnight sector, for instance at the IRT station, the predominant component of the disturbed SI field was still DL, but in early morning and afternoon stations, the principal component of the disturbed SI field was DP (subfield with two-pulse structure waveform), and we were able to detect a different behaviour depending on the magnetic local time between the stations of CMO and NAQ, where the DP component was reversed. We determined an estimated boundary for this different waveform between 9 and $15 \mathrm{~h}$ (magnetic local time).

Comparing the upper rows in Figure 13 we observe a different behaviour, where the waveform of SI changes. To determine more accurately the latitude where the waveform changes its sense, we present geomagnetic field, $X$ and $Y$ components, from IMAGE data set (Fig. 14). Thanks to the location of IMAGE stations, with approximately the same local time 
and covering a strategic area, we can detect when the two-pulse structure changes its behaviour, and as a consequence, we can determine where the boundary of the two ionospheric current systems, IC, is located. Regarding the $X$ component, in every observatory, we identify an outstanding double pulse structure which is due to the polar SI field disturbance, DP. There, we distinguish both the preliminary impulse and main impulse, PI and MI. For the PI impulse, stations poleward of SOR showed a positive impulse which indicates an eastward IC, and stations equatorward of TRO showed a negative impulse which indicates a westward IC. For the MI impulse, stations poleward of SOR showed a negative impulse indicating a westward IC, and stations equatorward of TRO showed a positive impulse which indicates an eastward IC. So we assumed the twin vortex was located around $67^{\circ}$, between the SOR and TRO stations.

As we commented above, we aim to find relationships between spacecraft and ground observations. In the same way that we found different polarizations among GOES 11 and GOES 10 and 12, the first located at morning side and the others located at afternoon side, we expected to have the same behaviour at ground. Looking at the magnetic signatures of different observatories, for example, Figure 13, we found that the polarization for observatories located in the morning sector is clockwise, the same as GOES 11, and observatories located in the afternoon sector have counter-clockwise polarization, as GOES 10 and 12. But, there is an area of transition. To show where is this transition area in Figure 15, we plotted the $X$ component of the magnetic field versus the $Y$ component for magnetic observatories located close to the meridian plane including the point of the first impact of the wave front to the magnetopause. Thus, the polarization as the temporal evolution of the magnetic vector appears. Observatories on the four left panels show a linear polarization and they are in the transition area and observatories on the right panels have a counter-clockwise polarization and they are in the afternoon side with respect to the first contact point in the magnetopause.

\section{Discussion}

As we commented in Section 2, the propagation of the compressional wave in the inner magnetosphere has been studied for many years. The difference between our study and the other studies cited in this manuscript mainly arises in the large number of spacecraft used in this study. Large number of spacecraft implies more accuracy in the determination of the shock front orientation and its propagation inside the magnetosphere. This work has some points in common with those of older studies, however in this section we want to point out the differences.

In the Introduction section, we cited Wilken et al. (1982) as a classical study about the propagation of the compressional wave front in the inner magnetosphere and how a sudden impulse wave front in the equatorial plane is deformed. Nowadays, taking advantage of the proliferation of different spacecraft, we can present a more realistic overview of the deformation of the SI wave front. That implied more redundancy and reliability in the determination of the orientation plane. Regarding the waveform deformation inside the magnetosphere, Wilken et al. (1982) draw a schematic representation of this deformation based on observations of spacecraft located in the afternoon side and early night side. With major number of spacecraft we covered the whole magnetosphere, and therefore we presented a more realistic representation of the waveform deformation.

We were interested in the complete overview of the propagation of the front wave of solar wind until its arrival on the Earth and the form of the SI at ground. To do this, we related spacecraft observations to a large number of waveforms observed on ground observatories, the different polarization between night and day sectors both at ground and at spacecraft' level shows the relation between these two data sets. This study is based on multipoint observation method, as other papers cited in the Introduction e.g. Keika et al. (2008, 2009), but they have other objectives than those in our study. Our representation of the wave front inside the magnetosphere is more realistic due to the major number of spacecraft available and therefore it is based more on observational facts. And we focused on magnetopause oscillations and the bow shock compression.

Recently, other studies, as those of Samsonov et al. (2011) or Volwerk et al. (2011), dealt with the wave propagation through the magnetosphere. They focused their attention on other topics as pulsation propagation or the response of the magnetosphere to $B_{z}$ changes. In this way, due to this extreme specialization, every study focuses on different topics, but all of them have in common the aim of examining the magnetospheric response to solar wind variations. The sum of these different studies finally allows us to have a really complete view of the response of the magnetosphere. In this work, we focused our attention only on the SI phenomena, obtaining a complete view from the solar wind variation to the Earth signatures.

The large number of spacecraft used in this study owes much to international collaboration and open data policy in Space Physics, which has become popular recently. This study indicates the importance of the open data policy for boosting research activity.

\section{Summary and conclusions}

This study provided a complete view of a sudden impulse, SI, from the enhancement of the solar wind pressure on its arrival at the magnetosphere to its magnetic signature on the ground. Using multipoint spacecraft observations and thanks to their particularly suitable location, we presented in detail how each part of the whole magnetosphere responds to a sudden enhancement of solar wind.

Our study case had a small propagation velocity and a small enhancement of solar wind pressure. Outside the magnetosphere, the propagation velocity of this disturbance was only $450 \mathrm{~km} / \mathrm{s}$, far below most of the other reported cases, whose velocity was around $600-900 \mathrm{~km} / \mathrm{s}$. Its dynamic pressure, $P_{\mathrm{SW}}$, was only $2 \mathrm{nPa}$, and it produced an increase of about $10 \mathrm{nT}$ in the magnetic field on the ground. Even with its small amplitude, this SI presented most of the typical signatures of these kinds of events, so we can conclude that the key aspect determining an SI was its suddenness more than its amplitude.

On the basis of IMF data from ACE, WIND and GEOTAIL spacecraft, we calculated the orientation of the solar wind front shock, obtaining an orientation of $(\phi, \theta)=\left(155^{\circ}, 2^{\circ}\right)$, where $\phi$ and $\theta$ are the longitude and latitude in GSM coordinates.

By means of THEMIS B and THEMIS C magnetosheath and bow shock observations at the moment of the SI event, we detected a collection of magnetopause crossings. Before the front shock crossing, with quiet conditions, we detected 
oscillations of the magnetopause from THEMIS C data, which were in concordance with magnetopause oscillations. The magnetic field in the magnetosheath, according to THEMIS $\mathrm{B}$ records, was larger than that recorded outside the magnetosphere, and had the same large-scale structure. After the shock front crossing, we identified the whole compression of the magnetosphere. Thus, these spacecraft could record magnetic features from other parts of the magnetosphere, in this way, THEMIS B recorded the interplanetary magnetic field, IMF, and THEMIS $\mathrm{C}$ recorded the magnetic field in the magnetosheath.

Using multipoint spacecraft data observation method, with the arrival time of the perturbation for each spacecraft, we calculated the solar wind front shock deformation inside the magnetosphere, obtaining great detail in the dayside area.

The ratio between the enhancement of magnetic field, $\Delta B$, and the square root of solar wind pressure, $R=\Delta B /\left(P_{2}^{1 / 2}-P_{1}^{1 / 2}\right)$ (Siscoe et al. 1968), on the ground was $R=15.5 \mathrm{nT} / \mathrm{nPa}^{1 / 2}$. This was consistent with other estimated values reported in the literature. In the geosynchronous orbit, $R$ showed a difference between GOES 11, located early morning, and GOES 10 and 12, both located in the afternoon. $R$ for GOES 10 and 12 is smaller than for GOES 11 . In addition, there was a clear difference between THEMIS A, located on the night side, and THEMIS D and THEMIS E, located at dawn. These observations revealed a large variety of amplitudes for the different time sectors, with the amplitudes being always smoother near the night sector.

The superposition of several magnetospheric and ionospheric contributions makes the manifestation of an SI on the ground more complex than in the geosynchronous orbit. In this study, we present a compilation of observations in which their waveforms were consistent with those of Araki's model (Araki 1994), in the model the disturbed field of SI is decomposed into two subfields, DL and DP, with different origins. Studying the pattern of the SI waveform from different observatories around the Earth, we identified where the SI waveform changes its features, which indicates different effects on the Earth produced by the ionospheric currents. Thanks to the IMAGE array of magnetometers, with a high density of stations, we were able to calculate the location of the boundary between these ionospheric current systems, only on the basic of waveform analysis. For our event, it was located around $67^{\circ}$ of geographical latitude.

Finally, we performed an analysis of polarization. We related polarization at ground to polarization measured by GOES spacecraft, and we also determined the longitudinal zone where polarization changes, what is consistent with the location of the first shock front arrival on the magnetopause.

Acknowledgements. This research has been supported by Spanish projects CTM2008-03033-E, CTM2009-13843-C02-01 and CTM2010-21312-C03-01 of MINECO. And supported by project 2014 SGR 175 of Generalitat de Catalunya, and the project 2014URL-Trac-039 of Ramon Llull University - Obra Social "la Caixa". The results presented in this paper rely on data collected at magnetic observatories. We thank the national institutes that support them and INTERMAGNET for promoting high standards of magnetic observatory practice (www.intermagnet.org). We also thank the institutes that maintain the IMAGE Magnetometer Array, Los Alamos National Laboratory spacecraft, THEMIS spacecraft and Cluster spacecraft. We thank NASA's National Space Science Data Center and Space Physics Data Facility for data usage. The editor thanks two anonymous referees for their assistance in evaluating this paper. This work was supported by Japanese International Exchange Program of National Institute of Information and Communications (NICT). This study was partly supported by the Ministry of Education, Culture, Sports, Science and Technology of Japan (MEXT), grant-in-aid for Scientific Research (B) (Grant 25287127).

\section{References}

Adams, W.G. Comparison of simultaneous magnetic disturbances at several observatories. Philos. Trans. R. Soc. London, Ser, 183, 131-140, 1892.

Akasofu, S.I., and S. Chapman. The sudden commencements of geomagnetic storms. URANIA, 44 (250), 321-358, 1959.

Araki, T. Global structure of geomagnetic sudden commencements, Planet. Space Sci., 25, 373-384, 1977.

Araki, T. A physical model of the geomagnetic sudden commencement. In: M.J., Engebretson, K. Takahashi, and M. Scholer, Editor. Solar Wind Sources of Magnetospheric UltraLow-Frequency Waves, Geophys. Monogr. Ser., vol. 81, AGU, Washington DC, 183, 1994.

Araki, T., T. Takeuchi, and Y. Araki. Rise time of geomagnetic sudden commencements - statistical analysis of ground geomagnetic data. Earth Planets Space, 56, 289-293, 2004.

Araki, T., K. Keika, T. Kamei, H. Yang, and S. Alex. Nighttime enhancement of the amplitude of geomagnetic sudden commencements and its dependence on IMF-Bz. Earth Planets Space, 58, 45-50, 2006.

Auster, H.U., K.H. Glassmeier, W. Magnes, O. Aydogar, W. Baumjohann, et al. The THEMIS fluxgate magnetometer. Space Sci. Rev., 141, 235-264, 2008, DOI: $10.1007 / \mathrm{s} 11214-008-9365-9$.

Balogh, A., M.W. Dunlop, S.W.H. Cowley, D.J. Southwood, J.G. Thomlinson, et al. The cluster magnetic field investigation. Space Sci. Rev., 79, 65-92, 1997.

Belian, R.D., G.R. Gisler, T. Cayton, and R. Christensen. High-z energetic particles at geostationary orbit during the great solar proton event series of October 1989. J. Geophys. Res., 97, 16897, 1992.

Carpenter, D.L., and R.R. Anderson. An ISEE/whistler model of equatorial electron-density in the magnetosphere. J. Geophys. Res., 97, 1097-1108, 1992, DOI: 10.1097/91JA01549.

Colburn, D.S., and C.P. Sonett. Discontinuities in the solar wind. Space Sci. Rev., 5, 43, 1966.

Curto, J.J., T. Araki, and L.F. Alberca. Evolution of the concept of Sudden Storm Commencements and their operative identification. Earth Planets Space, 59, i-xii, 2007.

Davis, T.N., and M. Sugiura. Auroral electrojet activity index AE and its universal time variations. J. Geophys. Res., 71 (3), 785-801, 1966, DOI: 10.1029/JZ071i003p00785.

Ellis, W. On the simultaneity of magnetic variations at different places on occasions of magnetic disturbance, and on the relation between magnetic and earth current phenomena. Proc. Roy. Soc., 102, 191, 1892.

Frank, L.A., K.L. Ackerson, W.R. Paterson, J.A. Lee, M.R. English, and G.L. Pickett. The Comprehensive Plasma Instrumentation (CPI) for the Geotail spacecraft. J. Geomagn. Geoelectr., 46, 23-37, 1994.

Iyemori, T. Storm-time magnetospheric currents inferred from midlatitude geomagnetic field variations. J. Geomagn. Geoelectr., 42, 1249-1265, 1990.

Keika, K., R. Nakamura, W. Baumjohann, A. Runov, T. Takada, et al. Response of the inner magnetosphere and the plasma sheet to a sudden impulse. J. Geophys. Res., 113, A07S35, 2008, DOI: $10.1029 / 2007$ JA012763.

Keika, K., R. Nakamura, W. Baumjohann, V. Angelopoulos, P.J. Chi, et al. Substorm expansion triggered by a sudden impulse front propagating from the dayside magnetopause. J. Geophys. Res., 114, A00C24, 2009, DOI: 10.1029/2008JA013445. 
A. Segarra et al.: Multipoint observation of the magnetosphere response to a SI event

Kokubun, S. Characteristics of storm sudden commencement at geostationary orbit. J. Geophys. Res., 88, 10025-10033, 1983.

Kokubun, S., T. Yamamoto, M.H. Acuña, K. Hayashi, K. Shiokawa, and H. Kawano. The Geotail magnetic-field experiment. J. Geomagn. Geoelectr., 46, 7-21, 1994.

Lepping, R.P., M.H. Acuña, L.F. Burlaga, W.M. Farrell, J.A. Slavin, et al. The Wind magnetic field investigation. Space Sci. Rev., 71, 207-299, 1995, DOI: 10.1007/BF00751330.

Lin, R.P., K.A. Anderson, S. Ashford, C. Carlson, D. Curtis, et al. A three-dimensional plasma and energetic particle investigation for the Wind spacecraft, Space Sci. Rev., 71, 125-153, 1995, DOI: $10.1007 /$ BF00751328.

Matsushita, S. Studies on sudden commencements of geomagnetic storms using IGY data from United States stations. J. Geophys. Res., 65, 1423-1435, 1960.

Rodés, L. On the non-simultaneity of magnetic storms. Terr. Mag. Atmos. Electr., 27 (4), 161-166, 1922.

Samsonov, A.A., D.G. Sibeck, N.V. Zolotova, H.K. Biernat, S.H. Chen, et al. Propagation of a sudden impulse through the magnetosphere initiating magnetospheric Pc5 pulsations. J. Geophys. Res., 116, A10216, 2011, DOI: 10.1029/2011JA016706.

Shue, J.H., P. Song, C.T. Russell, J.T. Steinberg, J.K. Chao, et al. Magnetopause location under extreme solar wind conditions. J. Geophys. Res., 103, 17691-17700, 1998.

Siscoe, G.L., V. Formisano, A.J. Lazarus. Relation between geomagnetic sudden impulses and solar wind pressure changes - an experimental investigation. J. Geophys. Res., 73, 15, 1968.

Smith, C.W., J. L'Heureux, N.F. Ness, M.H. Acuña, L.F. Burgala, and J. Scheifele. The ACE magnetic fields experiment. Space Sci. Rev., 86, 613-632, 1998, DOI: 10.1023/A:1005092216668.

Sugiura, M., and D.J. Poros. Hourly values of equatorial Dst for years 1957 to 1970, Rep. X-645-71-278, Goddard Space Flight Center, Greenbelt, Maryland, 1971.

Tanakade, A. Short preliminary report on three sudden commencements of geomagnetic storms. Comptes Rendues Assemblée de Lisbone, 1933, UGGI, Assoc. Magnetism Electr. Bull., 9, 149-157, 1934.
Tsunomura, S., and T. Araki. Numerical analysis of equatorial enhancement of geomagnetic sudden commencement. Planet. Space Sci., 32 (5), 599-604, 1984.

Tsurutani, B.T., and R.P. Lin. Acceleration of $>47 \mathrm{keV}$ ions and $>2$ $\mathrm{keV}$ electrons by interplanetary shocks at $1 \mathrm{AU}, J$. Geophys. Res., 90 (A1), 1-11, 1985.

Tsurutani, B.T., E. Echer, F.L. Guarnieri, and J.U. Kozyra. CAWSES November 7-8, 2004, superstorm: complex solar and interplanetary features in the post-solar maximum phase. Geophys. Res. Lett., 35, L06S05, 2008, DOI: 10.1029/2007GL031473.

Tsurutani, B.T., G.S. Lakhina, O.P. Verkhoglyadova, W.D. Gonzalez, E. Echer, et al. A review of interplanetary discontinuities and their geomagnetic effects. J. Atmos. Sol. Terr. Phys., 73, 5, 2011, DOI: $10.1016 /$ j.jastp.2010.04.001.

Tsurutani, B.T., E. Echer, K. Shibata, O.P. Verkhoglyadova, A.J. Mannucci, et al. The interplanetary causes of geomagnetic activity during the 7-17 March 2012 interval: a CAWSES II overview. J. Space Weather Space Clim., 4, A02, 2014, DOI: $10.1051 / \mathrm{swsc} / 2013056$.

Tsyganenko, N.A. A magnetospheric field model with a warped tail current sheet. Planet. Space Sci., 37, 5-20, 1989.

Volwerk, M., J. Berchem, Y.V. Bogdanova, O.D. Constantinescu, M.W. Dunlop, et al. Interplanetary magnetic field rotations followed from L1 to the ground: the response of the Earth's magnetosphere as seen by multi-spacecraft and ground-based observations. Ann. Geophys., 29, 1549-1569, 2011.

Vorotnikov, V.S., C.W. Smith, Q. Hu, A. Szabo, R.M. Skoug, et al. Automated shock detection and analysis algorithm for space weather application. Space Weather, 6, S03002, 2008, DOI: $10.1029 / 2007$ SW000358.

Wanliss, J.A., and K.M. Showalter. High-resolution global storm index: Dst versus YM-H. J. Geophys. Res., 111, A02202, 2006, DOI: $10.1029 / 2005 J A 011034$.

Wilken, B., C.K. Goertz, D.N. Baker, P.R. Higbie, and T.A. Fritz. The SSC on July 29, 1977 and its propagation within the magnetosphere. J. Geosphys. Res., 87, 5901-5910, 1982.

Cite this article as: Segarra A, Nosé M, Curto JJ \& Araki T. Multipoint observation of the response of the magnetosphere and ionosphere related to the sudden impulse event on 19 November 2007. J. Space Weather Space Clim., 5, A13, 2015, DOI: 10.1051/swsc/2015016. 